\title{
DifERENCIAS EN LA CONDUCTA DE EMPAREJAMIENTO ENTRE MUJERES JÓVENES UNIVERSITARIAS DE SANTIAGO DE CHILE EN RELACIÓN CON EL CONSUMO DE PÍLDORAS ANTICONCEPTIVAS ORALES*
}

\section{DifFERENCES IN MATING BEHAVIOR AMONG UNIVERSITY YOUNG WOMEN OF SANTIAGO DE CHILE IN RELATION TO THE CONSUMPTION OF ORAL CONTRACEPTIVE PILLS}

\author{
José Antonio Muñoz-Reyes ${ }^{* *}$, Pablo Polo***, Valeska Cid***, Giannina Buccioni****, \\ Ana MARÍA FERnÁNDEZ ${ }^{* * * * *}$, MICHELE DUFEY ${ }^{* * * * * * *}$, LUIS FLORES-PRADO********, \\ MARCIAL BELTRAMI ${ }^{* * * * * * * *}$ Y ENRIQUE TURIÉGANO*********
}

*Trabajo financiado con el Proyecto FONDECYT regular No 1140234 y el Proyecto FONDECYT postdoctorado N³160366 asignado al Dr. Pablo Polo.

**Doctor en Ciencias y Profesor de Biología. Investigador de Jornada Completa en el Centro de Estudios Avanzados de la Universidad de Playa Ancha. E-Mail: jose-munoz-cea@upla.cl

***Doctor en Psicobiología y Profesor de Biología. Investigador Postdoctoral en el Centro de Estudios Avanzados de la Universidad de Playa Ancha.

****Magister en Ciencias Biológicas, Profesora de Biología y Doctoranda en Neurociencias. Ayudante de Investigación en el Centro de Estudios Avanzados de la Universidad de Playa Ancha.

${ }^{* * * * \star L i c e n c i a d a ~ e n ~ E d u c a c i o ́ n ~ e n ~ B i o l o g i ́ a . ~ A y u d a n t e ~ d e ~ Z o o l o g i ́ a ~ e n ~ e l ~ D e p a r t a m e n t o ~ d e ~ B i o l o g i ́ a ~ d e ~ l a ~ U n i v e r s i d a d ~}$ Metropolitana de Ciencias de la Educación.

******Doctora en Psicología y Psicóloga. Académica de Jornada Completa en la Escuela de Psicología de la Universidad de Santiago de Chile.

${ }^{* * * * * * *}$ Doctora en Psicología y Psicóloga. Académica de Jornada Completa en la Facultad de Ciencias Sociales de la Universidad de Chile.

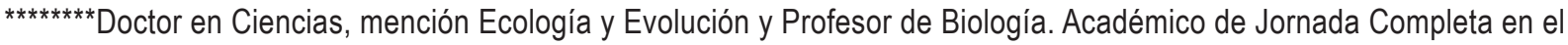
Instituto de Entomología de la Universidad Metropolitana de Ciencias de la Educación.

${ }^{* * * * * * * * * *}$ Magister en Ciencia y Profesor de Biología y Ciencias Naturales. Académico de Jornada Completa en el Departamento de Biología de la Universidad Metropolitana de Ciencias de la Educación.

*********Doctor en Ciencias y Biólogo. Académico de Jornada Completa en el Departamento de Biología de la Universidad Autónoma de Madrid.

Universidad de Playa Ancha. Valparaíso, Chile.

\section{RESUMEN}

Durante los últimos años en Chile se ha detectado una masificación en el uso de anticonceptivos orales. Sin embargo, en Chile y al igual que en casi todos los países latinoamericanos prácticamente no se han investigado sus implicancias sobre la conducta humana. Específicamente, este estudio evaluó el impacto del uso de estos anticonceptivos sobre la conducta de emparejamiento en un grupo de 164 mujeres jóvenes universitarias, con una media de edad igual a 19 años y provenientes de cuatro universidades chilenas. Para ello se utilizó la adaptación chilena del Cuestionario de Componentes del Valor de Pareja que está compuesto por 22 ítems que se responden en una escala Likert de siete valores. Los resultados señalan diferencias entre las mujeres que consumen píldoras anticonceptivas (PA) y las que no lo hacen. Las consumidoras presentan los mayores 
valores del índice general del cuestionario sobre los componentes del valor de pareja y las subescalas de historia de relaciones de pareja, apreciación desde el sexo opuesto y apariencia. Estos resultados indican que las mujeres consumidoras de la PA se autoperciben y creen que el sexo contrario las evalúa como atractivas y muestran una vida sexual más diversificada, no mostrando diferencias en los otros aspectos relevantes para el valor de pareja no vinculados centralmente al atractivo físico. En definitiva, los resultados parecen indicar que el consumo de anticonceptivos hormonales no modifica la conducta social y parental de las mujeres, aunque su uso parece estar asociado con una mayor autopercepción de atractivo y mayor diversidad sexual.

Palabras clave: Píldoras anticonceptivas; Valor de pareja; Mujeres jóvenes; Población chilena; Conducta de emparejamiento.

\section{ABSTRACT}

Oral contraceptives, also known as birth control pills (PAs from now on), have been available on the world market for five decades. At present, the use of PA is massifier worldwide, being consumed by more than 100 million women on the planet. In recent years, in Latin America has been detected a dramatic increase in the use of oral contraceptives, and Chile is not the exception. In this sense, it is probable that the increase in the consumption of PAs in Chile is linked to the increase of women who decide to pursue higher studies and to develop professional careers. Concurrently, a wide field of research has been focused on the physiological and psychological effect of the consumption of oral contraceptives on human behavior, being mating behavior one of the main topics of research. However, in Chile as in most Latin American countries there is a lack of studies on the implications of the consumption of oral contraceptives on human behavior. In the present study, we investigated in a group of 164 university young women (Mean \pm Standard Deviation: 19.27 \pm 2.88 years) of Chile the impact of the use of oral contraceptives on mating behavior. Accordingly, we have applied a validated psychometric test to assessed mate value, one of the more relevant components of mating behavior i.e., the Mate Value Questionnaire. Mate value can be defined as a construct that incorporates a number of physical, psychological, and social dimensions that are related to an individual's ability to find, attract, and successfully retain a partner. This questionnaire is composed by 22 items and 7 subscales that are answered in a Likert Scale of 7 values. The Mate Value Questionnaire showed a high reliability $(\alpha=.85)$. According with previous studies that showed a decrease in sexual satisfaction and desire on women that consume oral contraceptives, we predicted a decrease of mating behavior and, then, a decrease on Mate Value for those women that use oral contraceptives. This effect was expected for all subscales of this questionnaire with the exception of relationship history subscale since women that consume oral contraceptives reported to have more diverse sexual partners on previous studies. The results show differences between women who use oral contraceptives ( $n=87$ women: $19.60 \pm 2.89$ years) in comparison to those who do not $(n=77$ women: $18.90 \pm 2.84$ years). However, the differences were, in general, opposed to our expectations being the consumers of oral contraceptives who presented the highest values of the general index of the Mate Value Questionnaire, and, concretely, for the sub-scales of relationship history, views of the opposite sex, and looks. In addition, we have failed into found any differences between groups in subscales that were not associated to physical appearance, such as wealth, sociality and fear of failure. The results suggest that the use of hormonal contraceptives does not modify the social and parental behavior of the women, although their use seems to be associated with a greater self-perception of attractiveness and greater diversity in sexual life. This study provides new information that will increase the understanding and discussion about the hypothetical effect that would or would not, the consumption of the PA on the pairing behavior. It is important to point out that the field of research on changes in the sexual preferences of women, derived from hormonal states, is in an important discussion process. Therefore, we believe that it is critical to carry out research in the future in order to elucidate the real robustness of this phenomenon, improving our understanding about the behavioral effects of consuming oral contraceptives. 
Key words: Oral contraceptives; Mate value; Young women; Chilean population; Mating behavior.

\section{INTRODUCCIÓN}

Los anticonceptivos orales, también conocidos como píldoras anticonceptivas (PAs), están disponibles en el mercado mundial desde hace cinco décadas (Groves, Mosher, Lepkowski \& Kirgis, 2009). Actualmente, el uso de la PA se encuentra masificado a nivel mundial siendo consumidas por más de 100 millones de mujeres en el planeta (Alvergne \& Lummaa, 2010). El uso de la PA cambia el estado hormonal durante el ciclo menstrual simulando un embarazo, debido a que se inhibe la liberación de las hormonas folículo estimulante y luteinizante (Frye, 2006). Como consecuencia, se produce una disminución general de las hormonas producidas en los ovarios (Fleischman, Navarrete \& Fessler, 2010) y una suavización del peak de estrógenos que se produce a mitad de ciclo durante un proceso normal (van Heusden \& Fauser, 2002). Todo ello se traduce finalmente en una ausencia de maduración folicular, de ovulación y por tanto, del período fértil (Alvergne \& Lummaa, 2010). Según la Organización Mundial de la Salud este método anticonceptivo se sitúa en la cuarta posición de mayor uso considerando cualquier tipo de método anticonceptivo tras el coito interrumpido (método tradicional), el preservativo y el dispositivo intrauterino (DIU). En España se ha incrementado el consumo de la PA en mujeres en edad fértil de un $14.2 \%$ en el año 1997 a un 20.3\% en el año 2007 (Serrano, 2009). Por otra parte, en los países de América Latina también se ha observado un incremento en el uso de los anticonceptivos orales, por ejemplo en Venezuela en el año 2009, el 39\% de las mujeres en edad fértil consumían la PA, siendo el anticonceptivo más utilizado (R. Fernández et al., 2009). En Perú, la PA ocupa un tercer lugar ya que la consume el 9.1\% de las mujeres en edad fértil (Instituto Nacional de Estadística e Informática, 2015). En Bolivia y Colombia, alrededor de un $5 \%$ y un $10 \%$ respectivamente de mujeres en edad fértil la consumen (Aliaga, Jiménez \& Rodríguez, 2011). Finalmente, en la población chilena se ha producido una masificación en el consumo de la PA que sigue el patrón de los países europeos anteriormente señalados (INJUV, 2017). En este sentido, la PA es el segundo método anticonceptivo más utilizado después del preservativo en población joven, con un 31\% en el año 2015 (INJUV, 2017).

De acuerdo a Fenieux (2010), la razón más probable por la cual se ha masificado el consumo de la PA en Chile se fundamenta en el cambio sociocultural que han presentado las mujeres en las últimas décadas. Por un lado, después de la dictadura militar (entre los años 1973-1990) se abrió un período de debate en el que se replanteó y se cuestionó la legitimidad del concepto de sexualidad defendido por las antiguas instituciones, lo que condujo en 1996 a un enfrentamiento entre el Gobierno y la Iglesia Católica por la política en torno a la educación sexual (Araujo, 2005). A su vez y como consecuencia de las transformaciones sociales derivadas del cambio de régimen político, se observó un aumento de la fuerza laboral femenina, que en el 2013 alcanzó a un $48.3 \%$ en el trimestre octubre-diciembre (Instituto Nacional de Estadística, 2015) y por otra parte, se evidenció el incremento de mujeres con estudios superiores. Un ejemplo de esto es que en el año 2008, 6 de cada 10 egresados de educación superior eran mujeres (MINEDUC, 2008). Estos cambios han llevado a una modificación del comportamiento sexual, los valores y las normas que afectan tanto a los hábitos sexuales de los jóvenes como de los adultos, adelantando la edad de la primera experiencia sexual y su significado, incrementando la variedad de prácticas sexuales o retrasando la edad de matrimonio (Barrientos, 2006). Estos cambios han afectado de manera importante a las mujeres ya que la normalización de los métodos anticonceptivos y la mayor independencia adquirida en diferentes facetas de sus vidas, entre ellas la sexual, han llevado a que ellas decidan retrasar la maternidad y plani- 
ficar sus embarazos controlando su fecundidad principalmente con la PA (Fenieux, 2010). Actualmente, dos millones de mujeres en Chile consumen PAs, siendo la edad promedio de inicio del consumo a los 17 años; de hecho, estudios recientes han evidenciado que un $20.4 \%$ de la población femenina de 15 a 29 años de edad las utilizó en su primera relación sexual. Esta cifra aumenta al $73 \%$ en las jóvenes de estratos socioeconómicos medios y altos (INJUV, 2017). En este sentido, es notorio que los cambios socioculturales y la modificación del comportamiento sexual asociado a ellos no ha afectado de manera equivalente a todos los estratos sociales, de manera que la trasformación ha sido menos evidente en aquellos estratos sociales más desfavorecidos (Silva \& Barrientos, 2008). Consecuentemente, el consumo de la PA en Chile no es igualitario, ya que en mayor medida es utilizada por mujeres de 20 a 29 años, de un nivel socioeconómico alto (ABC1 y C2) y con estudios superiores universitarios (INJUV, 2017).

Si bien la planificación familiar y el uso de anticonceptivos es una necesidad social, la reducción de los niveles hormonales durante el ciclo ovárico producidos por la PA puede tener efectos sobre diferentes aspectos de la fisiología y comportamiento de las mujeres (Spencer, Bonnema \& McNamara, 2009). A este respecto, las consecuencias comportamentales y psicológicas derivadas del uso de PA han recibido mucha menor atención que las consecuencias físicas y fisiológicas; sin embargo, desde hace algunos años se ha desarrollado internacionalmente una línea de investigación para estudiar el efecto del consumo de las PA sobre el comportamiento (cf., Welling, 2013). Tal vez uno de los resultados más interesantes, polémicos y replicados de forma consistente a lo largo de diferentes poblaciones radica en el rol modificador de la PA sobre el comportamiento de emparejamiento femenino (cf., Alvergne \& Lummaa, 2010).

La variación hormonal que se produce de manera natural durante el ciclo ovárico está relacionada con un cambio en las preferencias de las mujeres por ciertos rasgos masculinos (cf., Gangestad \& Thornhill, 2008). De esta manera, diversos estudios muestran que las mujeres prefieren rasgos más masculinos y simétricos durante la fase fértil que durante la fase lútea de su ciclo ovárico (Havlicek, Roberts \& Flegr, 2005; Little, Jones \& DeBruine, 2008; Little, Jones \& Burriss, 2007; Penton-Voak \& Perrett, 2000).

Sin embargo, este cambio en las preferencias parece no producirse en las mujeres que consumen PA, lo que puede tener importantes consecuencias para la satisfacción sexual en mujeres emparejadas y con un uso discontinuado de la PA (Roberts et al., 2012; Roberts et al., 2014; Russell, McNulty, Baker \& Meltzer, 2014) y que en términos generales desencadenaría una aparente reducción del interés sexual (Bancroft, Sanders, Warner \& Loudon, 1987; Caruso et al., 2004; Graham, Ramos, Bancroft, Maglaya \& Farley, 1995; Sanders, Graham, Bass \& Bancroft, 2001; Wallwiener et al., 2010). A su vez, la variación hormonal que se produce durante un ciclo ovárico normal también influye en el atractivo de la propia mujer. Numerosos estudios encuentran que diversos rasgos como son la forma de la cara, el olor y la voz se vuelven más atractivos durante la fase fértil del ciclo, y además, estos cambios son percibidos por los hombres (Miller, Tybur \& Jordan, 2007; Pipitone \& Gallup, 2008; Roberts et al., 2004; Singh \& Bronstad, 2001). Junto a la variación en las preferencias por los rasgos masculinos, este incremento del atractivo de las mujeres durante la fase fértil del ciclo se piensa que funcionaría como una señal para atraer y retener con mayor eficacia a hombres con características indicadoras de buenos genes durante la etapa fértil de su ciclo (Alvergne \& Lummaa, 2010; Gangestad $\&$ Thornhill, 2008). Las mujeres que consumen PA no muestran esa variación de atractivo a lo largo de su ciclo, lo que podría disminuir su capacidad de competir con otras mujeres y de atraer y mantener eficazmente posibles parejas (Welling, 2013). De hecho, el consumo de la PA se ha vinculado a un aumento de la intensidad de la respuesta de celos (Geary, DeSoto, Hoard, Sheldon \& Cooper, 2001), especialmente para aquellos 
anticonceptivos con dosis más altas de estradiol (Cobey et al., 2012) y una disminución de la intensidad de la competencia intrasexual, aunque sólo en aquellas mujeres involucradas en una relación de pareja (Cobey, Klipping \& Buunk, 2013). Estos cambios podrían tener relación con la alteración de las señales y preferencias que sufren las mujeres que consumen PA, aunque no existe una evidencia directa clara. Sin embargo, los cambios negativos emocionales, de estado de ánimo general y de bienestar sexual son importantes predictores de la discontinuación en el uso de anticonceptivos orales (Graham et al., 1995; Rosenberg \& Waugh, 1998; Rosenberg, Waugh \& Meehan, 1995; Walker \& Bancroft, 1990), aunque esta sigue siendo un área en la que existen estudios con evidencia contradictoria, especialmente para la relación con el estado de ánimo y su variación a lo largo del ciclo (Caruso et al., 2005; Oinonen \& Mazmanian, 2002).

Por otro lado, el uso de la PA podría estar asociado a ciertos patrones sociales y comportamentales previos a su uso. A este respecto, se ha descrito una tendencia hacia una mayor diversidad de parejas sexuales y una mayor tendencia hacia la búsqueda de relaciones de corto plazo entre aquellas mujeres que consumen PA (Guillermo, Manlove, Gray, Zava \& Marrs, 2010; Little, Jones, Penton-Voak, Burt \& Perrett, 2002), si bien no se encuentra establecida claramente la relación causal entre ambas variables. Es decir, el deseo hacia mantener relaciones con un número diverso de parejas puede conducir al consumo de PA o bien el consumo de PA y la reducción de los costes asociados al sexo casual puede aumentar la diversidad de parejas sexuales.

En conjunto, los resultados presentados anteriormente destacan la importancia de investigar la conducta de emparejamiento femenina en las consumidoras de la PA, con el fin de caracterizar a este grupo, establecer diferencias y posibles problemáticas o beneficios, que pueden producirse como resultado del consumo de la PA. Dichas investigaciones resultan aún más importantes en la población chilena, debido a la ausencia de estudios so- bre el posible efecto psicológico del consumo de la PA sobre la conducta femenina y específicamente, la conducta de emparejamiento. De hecho, hasta la fecha no se ha realizado este tipo de investigaciones en poblaciones hispanoparlantes. Lo anterior es una alerta que denota la necesidad de generar datos cuantitativos que permitan a la Psicología mejorar y profundizar el conocimiento de este fenómeno, no solo utilizando los antecedentes derivados de población anglosajona. En este sentido, es claro que si los efectos de las PAs sobre el comportamiento humano tienen una fuerte base biológica, entonces estos deberían ser mensurables de manera ubicua en las diferentes poblaciones en el mundo. Sin embargo, los factores sociales e intrínsecos de cada cultura pueden atenuar o intensificar dichos efectos. Algo que no se puede saber sin tener evidencia intercultural como la que aporta este estudio.

Uno de los conceptos más relevantes para comprender la conducta de emparejamiento en la especie humana es el denominado valor de pareja (del inglés mate value - Fisher, Cox, Bennett \& Gavric, 2008). Este concepto se deriva desde la Psicología Evolucionista y se define como la suma total de características que posee un individuo en un momento dado dentro de un contexto particular y que impacta sobre su habilidad de encontrar, atraer y retener exitosamente a una pareja (Fisher et al., 2008). En este sentido, existe un Cuestionario de Valor de Pareja (CVP), diseñado por Fisher y colaboradores (2008) y recientemente validado en población chilena (Fernández, Muñoz-Reyes, Dufey, Buccioni \& Cid, 2015) que explora siete dimensiones diferentes de este constructo a nivel físico, psicológico y social. Estas dimensiones se refieren a la apreciación del sexo opuesto acerca de las habilidades sociales, el interés por la paternidad / maternidad, la importancia que le dan a exhibir su riqueza, la historia de relaciones previas, la importancia que le dan a su apariencia y sobre el miedo al fracaso.

El CVP ha sido evaluado en más de 30 investigaciones diferentes en el área de la sexualidad. Por ejemplo, en relación con la au- 
toestima (Goodwin et al., 2012), las estrategias de agresión intrasexual femenina (Fisher, Cox \& Gordon, 2009) o su vínculo con marcadores corporales de atractivo (Fernández, Muñoz-Reyes \& Dufey, 2014), etc. Dicha prueba psicométrica podría ser considerada como una importante herramienta para medir las diferencias en torno a la conducta de emparejamiento que pueda existir entre la población consumidora y no consumidora de PA. Sin embargo, como se ha mencionado anteriormente, los estudios previos únicamente han evaluado el efecto del consumo de PA en algunas de las dimensiones del valor de pareja y en concreto, sobre el atractivo físico (por ejemplo, Miller et al., 2007; Pipitone \& Gallup Jr, 2008), relacionado con la Subescala de Apariencia y sobre la diversidad de parejas (por ejemplo, Little et al., 2002), relacionada con la Subescala de Historia de Relaciones Previas.

Dado el número escaso de investigaciones en Chile en estos tópicos, a través de este estudio se pretendió caracterizar a una población de jóvenes universitarias chilenas en torno a su autopercepción del valor de pareja y establecer diferencias entre quienes consumen la PA y quienes no lo hacen.

Asumiendo la hipótesis de que el consumo de PA modifica el comportamiento de emparejamiento femenino eliminando la variación de atractivo a lo largo del ciclo ovárico y limitando la capacidad de atraer y retener a las parejas por parte de las consumidoras de PA, se podría esperar que las mujeres consumidoras de PA muestren globalmente una autopercepción del valor de pareja menor que las no consumidoras. Esas diferencias se espera verlas marcadas por una menor autoevaluación de las escalas físicas de Apariencia y Apreciación desde el sexo opuesto por parte de las consumidoras de PA. Si además se asume que la mayor diversidad de parejas que muestran las mujeres consumidoras de PA se deriva de una reducción de los costes del sexo casual y de un cambio comportamental en la orientación sociosexual, entonces se podría esperar que, a pesar de que ellas muestren una autopercepción del valor de pareja más bajo en general, a nivel de la Subescala de Historia de Relaciones, los valores deberían ser más elevados en comparación a las mujeres que no consumen PA.

\section{MÉTOdo}

\section{PARTICIPANTES}

La muestra estuvo integrada por $164 \mathrm{mu}-$ jeres heterosexuales jóvenes $(M \pm D E=19.27$ \pm 2.88 años) universitarias provenientes de cuatro casas de estudio de la Región Metropolitana de Chile.

Las universidades fueron seleccionadas siguiendo un criterio de conveniencia de la muestra. Se descartaron dos participantes homosexuales ya que no era posible articular las predicciones en torno a esta orientación sexual. Las universidades participantes acogen a estudiantes de niveles socioeconómicos medios y bajos principalmente, de hecho, todas están acogidas a la nueva Ley de Gratuidad en la Educación, que beneficia a los sectores socioeconómicos más vulnerables de la población.

Del total de mujeres, 87 eran consumidoras de la PA (19.60 \pm 2.89 años) y 77 no lo eran (18.90 \pm 2.84 años). Las participantes no recibieron ningún tipo de compensación económica o académica por participar.

\section{Procedimiento}

Las mujeres fueron invitadas a participar en el estudio a través de anuncios distribuidos en las cuatro universidades (es decir, carteles e invitaciones orales de los investigadores). Se solicitó a las interesadas que contactaran a un correo electrónico a través del cual se establecía una fecha para la aplicación del instrumento.

El estudio fue anónimo y contó con la aprobación del Comité de Bioética de la Universidad de Playa Ancha. Cada participante completó un consentimiento informado previo a la administración de los cuestionarios. Los instrumentos fueron aplicados al inicio del año lectivo. 


\section{INSTRUMENTO}

\section{Cuestionario de Valor de Pareja (CVP)}

En primer lugar las participantes cumplimentaron un breve cuestionario con preguntas generales (edad y orientación sexual). Además, se les pidió que indicaran si consumían anticonceptivos orales anovulatorios. En función de su respuesta se dividió la muestra en dos categorías: mujeres consumidoras de PAs y mujeres no consumidoras. A continuación, se les pidió que contestaran el CVP. Se utilizó la adaptación chilena (Fernández et al., 2015) del cuestionario de componentes del valor de pareja de Fisher y colaboradores (2008). Este cuestionario está compuesto por 22 ítems que se responden en una escala Likert de 7 valores $(1=$ discrepo fuertemente a $7=$ concuerdo fuertemente). El cuestionario abarca las siguientes dimensiones:

- Apreciación desde el sexo opuesto, que contiene seis ítems que evalúan la percepción del individuo sobre su propio éxito con el sexo opuesto.

- Habilidades sociales contiene cinco ítems que evalúan la sociabilidad del individuo.

- Cuidado de los hijos contiene tres ítems que evalúan la importancia que los individuos le dan a ser visto como un buen padre / madre y la intención de tener hijos.

- Dinero contiene dos ítems que evalúan las tendencias a mostrarse adinerado.

- Apariencia contiene dos ítems que evalúan la importancia que tiene el propio aspecto físico para el individuo.

- Historia de relaciones de pareja contiene dos ítems que evalúan la cantidad de parejas y la capacidad de atracción a nuevas parejas.

- Miedo al fracaso contiene dos ítems que evalúan el miedo de la persona a ser rechazada.

Cada dimensión se calculó de acuerdo a las indicaciones de Fisher y colaboradores (2008), es decir, sumando los puntajes de los ítems que la componen. Este estudio mostró una fuerte consistencia interna, evidenciada a partir del valor Alpha de Cronbach (.83), similar a la reportada en otros estudios (Chile: .85 y .83 en Fernández et al., 2014 y Fernán- dez et al., 2015, respectivamente; Canadá: .83 en Fisher et al., 2008).

\section{ANÁLISIS ESTADÍSTICOS}

Para evaluar las diferencias en el CVP entre los grupos de mujeres consumidoras de PA y no consumidoras se empleó una prueba $t$ para muestras independientes debido a la distribución normal de la variable. Sin embargo, la normalidad desapareció al comparar las diferentes subescalas del CVP, por lo que estas comparaciones se realizaron con la prueba $U$ de Mann Whitney. Los análisis se realizaron con el programa IBM SPSS 20, y el nivel de significación se estableció para un valor de Alpha igual a .05.

Para las diferencias significativas se calcularon además los intervalos de confianza para la diferencia de medias (para los datos no paramétricos se empleó el estadístico de Hodges-Lehmann para la mediana) y el tamaño del efecto. Para esto último se utilizó el estadístico $d$ de Cohen que fue calculado manualmente aplicando la fórmula habitual.

\section{Resultados}

Los estadísticos descriptivos se reportan en la Tabla 1. Siguiendo la predicción se analizó si existían diferencias significativas entre las mujeres que declararon consumir PAs y aquellas que no lo hacían, para el CVP y sus subescalas.

Se encontraron diferencias en el índice general del CVP $\left(t_{162}=2.52 ; p<.05\right.$; Intervalo de confianza para la diferencia de medias superior: 11.23, inferior: 1.36) (ver Figura 1 ), siendo las consumidoras de PAs quienes presentaron el valor de CVP más elevado (ver Tabla 1). Este tamaño del efecto fue moderado $(d$ de Cohen $=.4)$.

En las suescalas se encontraron diferencias significativas en Apreciación desde el sexo opuesto $(U=2581 ; p<.01$; Intervalo de confianza superior: 5, inferior: 1), Apariencia $(U=2486.50 ; p<.01$; Intervalo de confianza superior: 2, inferior: .00) e Historia de rela- 
ciones de pareja $(U=2661 ; p<.05$; Intervalo de confianza superior: 2 , inferior: .00). Los tamaños de efecto fueron moderados para Apreciación desde el sexo opuesto $(d=.4)$, Apariencia $(d=.4)$ e Historia de relaciones de pareja $(d=.3)$. En todas ellas, el grupo de consumidoras de PAs presentó valores más elevados que las que no consumían PAs (ver Tabla 1).

Por otro lado, no se encontraron diferencias significativas entre ambos grupos para las siguientes dimensiones: Cuidado de los hijos $(U=2915 ; p=.151)$, Dinero $(U=3122.5$; $p=.406)$, Habilidades sociales $(U=3202$; $p=.627)$ y Miedo al fracaso $(U=3104$; $p=.416)$.

\section{Discusión}

La sociedad chilena es habitualmente definida como conservadora en aspectos sexuales y reproductivos, no obstante, durante la última década se ha vivido una apertura paulatina en torno a la discusión sobre sexualidad y derechos reproductivos (Macintyre, Montero Vega \& Sagbakken, 2015). Es probable que el aumento del consumo de PAs en Chile esté vinculado a esta mayor apertura social y más aún, al incremento de las mujeres que deciden cursar estudios superiores y desarrollar carreras profesionales (Fenieux, 2010; INJUV, 2017). Sin embargo, aunque la inhibición de la ovulación y los cambios hormonales necesarios para ello se han asociado a diversos efectos fisiológicos, comportamentales y psicológicos (Graham et al., 1995; Spencer et al., 2009; Welling, 2013), lamentablemente dichos efectos no han sido investigados previamente en tal población. En este estudio se investigó la relación entre el uso de las PAs y el comportamiento de emparejamiento de las mujeres chilenas a través de su efecto en la autopercepción del valor de pareja. El valor de pareja es un constructo que incorpora una serie de dimensiones físicas, psicológicas y sociales que están relacionadas con la habilidad de un individuo para encontrar, atraer y retener exitosamente a una pareja (Fisher et al., 2008) y que, por tanto, tienen importancia en el comportamiento de emparejamiento. Los resultados muestran diferencias en general en el valor de pareja entre los grupos, así como en algunas de las dimensiones de dicha variable. Sin embargo, a excepción de la Subescala Historia de las relaciones de pareja, los resultados obtenidos no están en línea con las predicciones que se plantearon ya que muestran un patrón opuesto al esperado. Aun así, constituyen evidencia que aporta al conocimiento y caracterización de la conducta de emparejamiento de las mujeres jóvenes consumidoras de la PA.

En esta investigación se esperaba que la autopercepción del valor de pareja se viese reducida en aquellas mujeres consumidoras de la PA al inhibirse en ellas el período fértil y modificar de esta manera el cambio en las preferencias y en el atractivo que las mujeres que ovulan normalmente presentan durante el ciclo ovárico (Welling, 2013).

Más concretamente, era de esperar que las subescalas relacionadas con los componentes físicos, es decir, las de Apreciación desde el sexo opuesto y Apariencia, estuviesen reducidas en las consumidoras de PAs, ya que se postula que la falta de incremento en el atractivo durante el período fértil que sufren estas mujeres limitaría su capacidad de atraer y retener parejas (Welling, 2013) y eso podría afectar a su autopercepción del valor de pareja. Sin embargo, los resultados obtenidos no apoyan dichas predicciones. En primer lugar, los valores generales de valor de pareja son mayores para aquellas mujeres que tomaron PA en comparación con las que no. En relación con la Subescala Apreciación desde el sexo opuesto, los resultados muestran que las mujeres que consumen la PA perciben que son vistas como más atractivas por los hombres. Esto conllevaría una mayor seguridad en sí mismas, ya que esta escala ha sido considerada como una medida de autoestima vinculada a la conducta de emparejamiento (Fisher et al., 2008). De hecho, esta dimensión se encuentra estrechamente asociada a la Escala de Autoestima General de Rosenberg (1973) (Fernández et al., 2015). Asimismo, en relación con la Subescala Apariencia, las mujeres consumidoras de PA se sienten y perci- 
ben como más atractivas que sus pares. A este respecto, existe cierta evidencia previa de dicha asociación entre consumo de la PA y el atractivo autopercibido (Clark, 2004).

Por otro lado, en la Subescala Historia de relaciones de pareja se encontró apoyo para nuestra predicción. Estudios previos han reportado que las mujeres que empleaban la PA manifestaban haber tenido mayor diversidad de parejas sexuales y una tendencia hacia la búsqueda de relaciones de corto plazo (Guillermo et al., 2010; Little et al., 2002). Los resultados obtenidos apuntan en la misma dirección, ya que las mujeres que consumen PA estarían reportando una historia de relaciones de pareja más diversa. Sin embargo, ni nuestros análisis ni los publicados en el estudio original de Little y colaboradores (2002) pueden entregar una valoración causal a esta relación, es decir, que el consumo de PA conllevaría un cambio psicológico que aumentaría el número de parejas sexuales. De hecho, si se tiene en cuenta la relación positiva entre el consumo de PA y las subescalas Apariencia y Apreciación desde el sexo opuesto parece más probable pensar que estas diferencias están dadas por diferencias previas en la orientación socio-sexual entre mujeres que consumen PA y las que no. De esta manera, las que se perciben como más atractivas e interesadas en relaciones a corto plazo consumirían anticonceptivos orales al tener un mayor acceso a parejas sexuales ya que saben que son especialmente valoradas por el sexo contrario. Por lo tanto, ellas estarían demostrando que son conscientes de la relevancia de su uso para evitar embarazos no deseados, lo que en última instancia daría cuenta de la aplicación de conocimientos en torno a educación sexual. En cualquier caso, son necesarios estudios futuros más profundos y de corte longitudinal para explorar la profundidad de esta relación entre estas tres subescalas con el consumo de PA.

En las restantes subescalas no se encontraron diferencias significativas entre las mujeres consumidoras de la PA y las que no las consumen, lo que indicaría que estas diferencias en el comportamiento no son extrapolables más allá de una dimensión física, ya que otros aspectos que influyen en el valor de la pareja, pero no relacionados directamente con el atractivo o apariencia física, no son diferentes entre grupos. De esta manera no se encontraron diferencias en la inversión parental entre los grupos, lo que impide concluir que aquellas mujeres que consumen PA busquen más que las no consumidoras retardar o impedir la maternidad. Tal vez la principal razón de esta falta de diferencias entre grupos, podría estar sustentada en la muestra en la cual se focalizó esta investigación, es decir, jóvenes mujeres universitarias. Ellas se encuentran en una etapa de desarrollo en la que la maternidad normalmente se retrasa conscientemente (Fenieux, 2010), ya sea a través del consumo de la PA, la abstinencia sexual $\mathrm{u}$ otros métodos anticonceptivos. De esta forma, se puede especular que las que no consumen la PA, probablemente están eligiendo otro método anticonceptivo, ya que no parecen particularmente interesadas en tener hijos.

Por último, tampoco se encontraron diferencias entre las subescalas Habilidades sociales, Dinero y Miedo al fracaso, por lo que los resultados obtenidos sugieren que las principales diferencias encontradas en torno a la conducta de emparejamiento entre grupos de mujeres consumidoras de PA versus no consumidoras sólo se encuentran en aquellos componentes que están íntegramente relacionados con el atractivo físico y no por ejemplo, con aspectos tan relevantes para la búsqueda de pareja como el desarrollo de habilidades sociales que es un rasgo de la personalidad atractivo para el sexo opuesto.

Se puede mencionar que el estudio realizado presenta ciertas limitaciones relevantes que deben ser señaladas. De esta forma, la población que afirmó estar consumiendo anticonceptivos orales no detalló el tipo de principio activo que tienen las píldoras. Por lo tanto, tal y como lo señalan los estudios en torno a la respuesta de celos en función del consumo de diferentes tipos de PA (por ejemplo, Cobey et al., 2012), podrían existir diferencias que surgirían a partir de las hormonas utilizadas y las concentraciones de las mismas que no fueron evaluadas en el presente estu- 
dio. Sin embargo, esta variable no suele ser considerada en estudios de este tipo (MuñozReyes et al., 2014; Penton-Voak et al., 1999) porque lo que se intenta establecer es la presencia de un efecto general derivado de la supresión de la fertilidad, independientemente de las concentraciones hormonales.

Por otro lado, al no ser longitudinal este estudio, se carece de información para una misma mujer sobre su autopercepción del valor de pareja en las dos condiciones, con consumo de PA y sin consumo, lo que sería valioso para evaluar las posibles diferencias previas en el valor de pareja de futuras consumidoras de PA con respecto a las que nunca las han consumido y establecer una relación causal entre el consumo de PA y los valores en la autopercepción del valor de pareja. Por último, aquellas mujeres que declararon no consumir PA podrían estar consumiendo otro tipo de anticonceptivo hormonal no oral ya que no se les preguntó sobre ello. Sin embargo, la tasa de consumo de anticonceptivos hormonales no orales es baja entre la población joven y universitaria. Por ejemplo, según la octava encuesta del Instituto Nacional de la Juventud, los parches transdérmicos fueron utilizados por el $3.4 \%$ de las mujeres mientras que las inyecciones anticonceptivas alcanzaron el 7.4\% (INJUV, 2017). A su vez, la posible inclusión de mujeres que emplean anticonceptivos hormonales no orales en el grupo de no consumidoras de PA no explicaría nuestros resultados, ya que en todo caso dificultaría la detección de diferencias entre los grupos.

Es importante señalar que pese a que se realizó una campaña de reclutamiento de participantes, el tamaño de la muestra fue relativamente modesto. En este sentido, existe evidencia de estudios previos en temáticas de sexualidad en los que se ha logrado un buen número de participantes (sobre 500 individuos en: Comisión Nacional del Sida, 2000; Fétis et al., 2008; González, Molina, Montero, Martínez \& Leyton, 2007; González, Molina, Montero, Martínez \& Molina, 2009; González, Molina \& San Martín, 2016; Schiappacasse, Bascuñan, Frez \& Cortés, 2014). Sin embargo, en estas investigaciones, la me- todología de recolección de datos, la promoción y el acceso a la muestra son diferentes. De esta forma, usualmente se aplican entrevistas cara a cara, auspiciadas por organismos del estado y con muestras cautivas, ya sean estudiantes de colegios o pacientes de hospitales. En contraposición, los estudios de reclutamiento abierto en torno a temas de sexualidad alcanzan tamaños muestrales muy similares a los de este estudio (por ejemplo, $n=117$ en Cárdenas \& Barrientos, 2008; $n=232$ en Conejero \& Almonte, 2009; $n=110$ en Gómez \& Barrientos, 2012), siendo menores a otros estudios con similar metodología pero que abordan características psicológicas no relacionadas directamente con la sexualidad (por ejemplo, $n=371$ en Fernández, Martínez-Conde \& Melipillán, 2009; $n=473$ en Rojas-Barahona, Zegers \& Förster, 2009). El equipo de investigación presupone que, pese a la apertura en temas sexuales que vive el país, el contexto conservador con el que aun este tipo de problemáticas son enfrentadas en la población chilena, juega un rol central. Como señalan otros autores (por ejemplo. Lehrer, E.L., Lehrer, V.L. \& Krauss, 2009; Morán Faúndes, 2013a), Chile es un país que se ha caracterizado por un férreo control conservador de sus políticas públicas de sexualidad y reproducción, derivado principalmente desde la Iglesia Católica. Ejemplos de esto son la difícil aprobación de la píldora anticonceptiva de emergencia, que recién ocurrió el año 2010 (Morán Faúndes, 2013b), la ilegalidad del aborto en cualquier tipo de circunstancia, incluyendo la violación o la inviabilidad del feto (Amnistía Internacional, 2015) y la legalización del divorcio en el año 2004 (Blofield, 2006). Esta política conservadora probablemente ha retrasado la aparición de estudios robustos en esta área que permitan tener líneas de base sobre la realidad país, incluso en temas de alta sensibilidad social. Un ejemplo de esto último son los recientes estudios en torno a los abusos sexuales en estudiantes universitarios (Schuster, Krahé, Ilabaca Baeza \& MuñozReyes, 2016) y la discriminación contra las minorías sexuales (Barrientos \& Bozon, 2014). Es por esto que esta investigación es 
parte de un conjunto de estudios que utilizando un perfil cuantitativo, intentan comenzar a generar resultados en un área de difícil acceso dentro de la política conservadora del país. De ahí que sumado a los resultados obtenidos, exista también una valoración cualitativa en torno al robustecimiento del área y a la generación de antecedentes que permitan discusiones más informadas.

En conclusión, esta investigación ha arrojado nuevos resultados que en su mayoría se contraponen a nuestras hipótesis iniciales basadas en la evidencia empírica reciente. Esta evidencia previa indica que las mujeres consumidoras de la PA no mostrarían los cambios en las preferencias por los rasgos masculinos $\mathrm{y}$ el incremento en su atractivo durante la etapa fértil que exhiben las mujeres que no consumen PA, lo que limitaría su habilidad en atraer y retener parejas (por ejemplo, Alvergne \& Lummaa, 2010; Welling, 2013) impactando negativamente sobre su autopercepción de su valor de pareja.

Sin embargo, en este estudio las mujeres consumidoras de la PA se autoperciben y creen que el sexo contrario las evalúa como atractivas y muestran una vida sexual más di- versificada, no mostrando diferencias en los otros aspectos relevantes para el valor de pareja no vinculados al atractivo físico.

En definitiva, los resultados parecen indicar que el consumo de anticonceptivos hormonales no modifica la conducta social y parental de las mujeres, aunque su uso parece estar asociado con una mayor autopercepción de atractivo y mayor diversidad en la vida sexual. Este estudio aporta nueva información que permitirá aumentar la comprensión y discusión sobre el hipotético efecto que tendría o no, el consumo de la PA sobre la conducta de emparejamiento. Es importante señalar que el campo de investigación en torno a los cambios en las preferencias sexuales de las mujeres, derivados de los estados hormonales, se encuentra en un importante proceso de discusión, ya que existe evidencia contrapuesta en torno a sus principales resultados (por ejemplo, Harris, Chabot \& Mickes, 2013; MuñozReyes et al., 2014). Por tanto, creemos que es crítico realizar investigaciones en el futuro con el fin de dilucidar la real robustez de este fenómeno, no solo en población anglosajona, sino también incluyendo nuevas poblaciones como la que ha participado en este estudio. 
Muñoz-Reyes, Polo, Cid, Buccioni, Fernández, Dufey, Flores-Prado, Beltrami y Turiégano

TABLA 1

Estadísticos descriptivos (Media \pm DEsViación estándar) PARA el Cuestionario de Valor de Pareja Y SUS SUBESCALAS

\begin{tabular}{|l|c|c|c|}
\hline \multicolumn{1}{|c|}{ Variable } & $\begin{array}{c}\text { Consumidoras PA } \\
(n=87)\end{array}$ & $\begin{array}{c}\text { No consumidoras } \\
(n=77)\end{array}$ & $\begin{array}{c}\text { Total } \\
(N=164)\end{array}$ \\
\hline Cuestionario de valor de pareja & $93.48 \pm 16.08$ & $87.18 \pm 15.83$ & $90.52 \pm 16.22$ \\
Apreciación desde el sexo opuesto & $27.79 \pm 6.27$ & $25.01 \pm 6.78$ & $26.48 \pm 6.64$ \\
Habilidades sociales & $21.28 \pm 5.98$ & $20.67 \pm 6.62$ & $21.00 \pm 6.28$ \\
Cuidado de los hijos & $15.59 \pm 4.49$ & $14.87 \pm 4.28$ & $15.25 \pm 4.39$ \\
Dinero & $3.47 \pm 2.09$ & $3.05 \pm 1.60$ & $3.27 \pm 1.88$ \\
Apariencia & $9.89 \pm 3.23$ & $8.62 \pm 3.15$ & $9.29 \pm 3.25$ \\
Historia de relaciones de pareja & $9.81 \pm 2.39$ & $8.87 \pm 2.74$ & $9.37 \pm 2.60$ \\
Miedo al fracaso & $5.62 \pm 2.65$ & $6.07 \pm 3.04$ & $5.83 \pm 2.84$ \\
\hline
\end{tabular}

FIGURA 1

MEDIA \pm DESVIACIÓN ESTÁNDAR PARA EL VALOR DE PAREJA PARA CONSUMIDORAS Y NO CONSUMIDORAS DE LA PÍLDORA ANTICONCEPTIVA (PA)

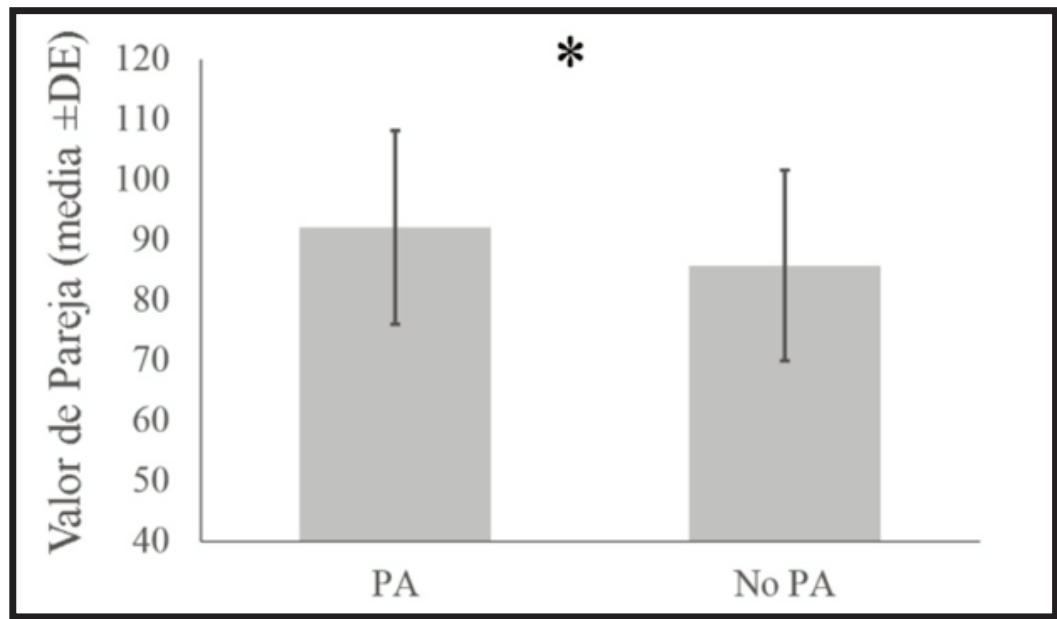

${ }^{*} p<.05$ 


\section{REFERENCIAS BIBLIOGRÁFICAS}

Aliaga, L., Jiménez, M. \& Rodríguez, J. (2011). Una mirada desde América Latina y el Caribe al objetivo de desarrollo del milenio de acceso universal a la salud reproductiva [A look from Latin America and the Caribean toward the developmental aim from the Millenium of reproductive health universal access]: CEPAL. Recuperado el 18 de julio de 2017 de http://reposito rio.cepal.org/bitstream/handle/11362/7132/S1 001054 es.pdf?sequence $=1$.

Alvergne, A. \& Lummaa, V. (2010). Does the contraceptive pill alter mate choice in humans? Trends in Ecology \& Evolution, 25(3), 171-179. http://dx.doi.org/10.1016/j.tree.2009.08.003

Amnistía Internacional. (2015). Amnesty International Report 2014/15: The State of the world's human rights. Madrid, España. Recuperado el 18 de julio de 2017 de https://www.amnesty.or g/en/documents/pol10/0001/2015/en/

Araujo, K. (2005). Sobre ruidos y nueces: Debates chilenos en torno a la sexualidad [On noise and nuts: Chilean debates on sexuality]. Iberoamericana, 5(18), 109-125.

Bancroft, J., Sanders, D., Warner, P. \& Loudon, N. (1987). The effects of oral contraceptives on mood and sexuality: A comparison of triphasic and combined preparations. Journal of Psychosomatic Obstetrics \& Gynecology, 7(1), 1-8. http://dx.doi.org/10.3109/01674828709019585

Barrientos, J. (2006). ¿Nueva normatividad en el comportamiento sexual juvenil en Chile? [New social rules on youth sexual behavior in Chile?]. Ultima Década, 14(24), 81-97. http://dx.doi. org/10.4067/S0718-223620060001000 05

Barrientos, J. \& Bozon, M. (2014). Discrimination and victimization against gay men and lesbians in Chile: Two patterns or just one? Interdisciplinaria, 31(2), 323-340. Recuperado el 18 de julio de 2017 de http://www.scielo.org. ar/sciel o.php?script=sci_arttext\&pid=S1668-7027201 4000200008\&lng=es\&tlng=en; https://doi.org/ 10.16 88 8/interd.2014.31.2.8

Blofield, M. (2006). The politics of moral sin: abortion and divorce in Spain, Chile and Argentina. New York, NY: Routledge.

Cárdenas, M. \& Barrientos, J. (2008). Actitudes explícitas e implícitas hacia los hombres homosexuales en una muestra de estudiantes universitarios en Chile [Explicit and implicit attitudes towards homosexual men in a sample of university students in Chile]. Psykhe (Santiago), 17 (2), 17-25. http://dx.doi.org/10.4067/S0718-22 282008000200002

Caruso, S., Agnello, C., Intelisano, G., Farina, M., Di Mari, L. \& Cianci, A. (2004). Sexual behavior of women taking low-dose oral contraceptive containing $15 \mu \mathrm{g}$ ethinylestradiol $/ 60 \mu \mathrm{g}$ gestodene. Contraception, 69(3), 237-240. http:// dx.doi.org/10.1016/j.contraception.2003.11.00 1

Caruso, S., Agnello, C., Intelisano, G., Farina, M., Di Mari, L., Sparacino, L. \& Cianci, A. (2005). Prospective study on sexual behavior of women using $30 \mu \mathrm{g}$ ethinylestradiol and $3 \mathrm{mg}$ drospirenone oral contraceptive. Contraception, 72(1), 19-23. http://dx.doi.org/10.1016/j.contraceptio n.2005.02.002

Clark, A.P. (2004). Self-perceived attractiveness and masculinization predict women's sociosexuality. Evolution and Human Behavior, 25 (2), 113-124. http://dx.doi.org/10.1016/S10 905138(03)00085-0

Cobey, K.D., Buunk, A.P., Roberts, S.C., Klipping, C., Appels, N., Zimmerman, Y. et al. (2012). Reported jealousy differs as a function of menstrual cycle stage and contraceptive pill use: A within-subjects investigation. Evolution and Human Behavior, 33(4), 395-401. http://dx.doi. org/10.1016/j.evolhumbehav.2011.12.001

Cobey, K.D., Klipping, C. \& Buunk, A.P. (2013). Hormonal contraceptive use lowers female intrasexual competition in pair-bonded women. Evolution and Human Behavior, 34(4), 294-298. 
http://dx.doi.org/10.1016/j.evolhumbehav.013. 04.003

Comisión Nacional del Sida. (2000). Estudio nacional de comportamiento sexual [National study of sexual behavior]. Santiago, Chile. Recuperado el 18 de julio de 2017 de http://www. scielo.cl/pdf/rmc/v144n2/art06.pdf

Conejero, J. \& Almonte, C. (2009). Desarrollo de la orientación sexual en adolescentes de 16 a 18 años de ambos sexos de Santiago de Chile: Estudio exploratorio [Development of sexual orientation in adolescents between 16 and 18 years of age in both sexes of Santiago de Chile: Exploratory study]. Revista Chilena de Neuropsiquiatría, 47(3), 201-208. http://dx.doi.org/ 10.4067/S0717-92272009000300004

Fenieux, C.G. (2010). Impacto social y psicológico de la píldora anticonceptiva [Social and psychological impact of the contraceptive pill]. Revistas de Obstetricia y Ginecología Hospital Santiago Oriente Dr. Luis Tisné Brousse, 5, 127-130.

Fernández, A.M., Muñoz-Reyes, J. A. \& Dufey, M. (2014). BMI, age, mate value, and intrasexual competition in Chilean women. Current Psychology, 33(4), 435-450. http://dx.doi.org/10. 1007/s12144-014-9221-x.

Fernández, A.M., Muñoz-Reyes, J.A., Dufey, M., Buccioni, G. \& Cid, V. (2015). Adaptación del cuestionario de componentes del valor de pareja al contexto chileno [Adaptation of the mate value questionnaire to the Chilean context]. $R e$ vista Iberoamericana de Diagnóstico y Evaluación Psicológica, 40(1), 94-102.

Fernández, O.M., Martínez-Conde, M. \& Melipillán, R. (2009). Estrategias de aprendizaje y autoestima: Su relación con la permanencia y deserción universitaria [Learning and self-esteem strategies: their relationship with university permanence and withdrawal]. Estudios Pedagógicos (Valdivia), 35(1), 27-45. http:// dx.doi.org/10.4067/S0718-0705200900 0100 002
Fernández, R., López, L., Martínez, H., Kopecky, D., Uzcátegui, G. \& Muñoz, M. (2009). Métodos anticonceptivos: Prevalencia de uso según método en el Hospital Materno Infantil del Este Petare [Contraceptive methods: Prevalence of use according the method in the Hospital Materno Infantil del Este Petare]. Revista de Obstetricia y Ginecología. Venezuela, 69(1), 28-34.

Fétis, G., Bustos, L., Lanas, F., Baeza, B., Contreras, J., Hebel, E. \& Marucich, C. (2008). Factores asociados al uso de anticonceptivos en estudiantes de enseñanza media de la comuna de Temuco [Factors associated with the use of contraceptives in middle school students in the commune of Temuco]. Revista Chilena de Obstetricia y Ginecología, 73(6), 362-369. http:// dx.doi.org/10.4067/S0717-752620080 006000 02

Fisher, M., Cox, A., Bennett, S. \& Gavric, D. (2008). Components of self-perceived mate value. Journal of Social, Evolutionary, and Cultural Psychology, 2(4), 156-168. http://dx.doi. org/10.1037/h0099347.

Fisher, M., Cox, A. \& Gordon, F. (2009). Selfpromotion versus competitor derogation: The influence of sex and romantic relationship status on intrasexual competition strategy selection. Journal of Evolutionary Psychology, 7(4), 287-308. http://dx.doi.org/ 10.1556/jep.7.2009. 4.6

Fleischman, D.S., Navarrete, C.D. \& Fessler, D. M. T. (2010). Oral contraceptives suppress ovarian hormone production. Psychological Science, 21 (5), 750-752. http://dx.doi.org/10.1177/095679 7610368062

Frye, C.A. (2006). An overview of oral contraceptives: Mechanism of action and clinical use. Neurology, 66(66 suppl 3), S29-S36. http://dx. doi.org/10.1212/WNL.66.66_suppl_3.S29

Gangestad, S.W. \& Thornhill, R. (2008). Human oestrus. Proceedings of the Royal Society B: Biological Sciences, 275(1638), 991-1000. http: //dx.doi.org/10.1098/rspb.2007.1425 
Geary, D.C., DeSoto, M.C., Hoard, M.K., Sheldon, M.S. \& Cooper, M.L. (2001). Estrogens and relationship jealousy. Human Nature, 12(4), 299-320. http://dx.doi.org/10.1007/s12110-0011001-2

Gómez, F. \& Barrientos, J. (2012). Efectos del prejuicio sexual en la salud mental de gays y lesbianas, en la ciudad de Antofagasta, Chile [The effects of sexual prejudice on the mental health of gays and lesbians in Antofagasta, Chile]. Sexualidad, Salud y Sociedad-Revista Latinoamericana, (10), 100-123.

González, E., Molina, T., Montero, A., Martínez, V. \& Leyton, C. (2007). Comportamientos sexuales y diferencias de género en adolescentes usuarios de un sistema público de salud universitario [Sexual behavior and gender differences among adolescents users of a public university health system]. Revista Médica de Chile, 135 (10), 1261-1269. http://dx.doi.org/10.4067/S 0034-98872007001000005

González, E., Molina, T., Montero, A., Martínez, V. \& Molina, R. (2009). Factores asociados a la continuidad del uso anticonceptivo en adolescentes solteras nulíparas [Factors associated with maintenance of contraception among adolescents]. Revista Médica de Chile, 137(9), 1187-1192. http://dx.doi.org/10.4067/S003498872009000900007

González, E., Molina, T. \& San Martin, J. (2016). Comportamientos sexuales y características personales según orientación sexual en adolescentes chilenos [Sexual behaviors and personal characteristics related to sexual orientation in Chilean adolescents]. Revista Chilena de Obstetricia y Ginecología, 81(3), 202-210. http:// dx.doi.org/10.4067/S0717-7526201600030006

Goodwin, R., Marshall, T., Fülöp, M., Adonu, J., Spiewak, S., Neto, F. \& Hernández Plaza, S. (2012). Mate value and self-esteem: Evidence from eight cultural groups. PLoS One, 7(4), e36106. http://dx.doi.org/10.1371/journal.pon e.0036106
Graham, C.A., Ramos, R., Bancroft, J., Maglaya, C. \& Farley, T.M.M. (1995). The effects of steroidal contraceptives on the well-being and sexuality of women: A double-blind, placebocontrolled, two-centre study of combined and progestogen-only methods. Contraception, 52 (6), 363-369. http://dx.doi.org/10.1016/00107824(95)00226-X

Groves, R.M., Mosher, W. D., Lepkowski, J.M. \& Kirgis, N.G. (2009). Planning and development of the continuous National Survey of Family Growth. Vital and Health Statistics. Ser. 1, Programs and Collection Procedures, 1(48), 1-64.

Guillermo, C.J., Manlove, H. A., Gray, P.B., Zava, D.T. \& Marrs, C.R. (2010). Female social and sexual interest across the menstrual cycle: The roles of pain, sleep and hormones. BMC Women's Health, 10(1), 19-29. http://dx.doi.org/ 10.1186/1472-6874-10-19

Harris, C.R., Chabot, A. \& Mickes, L. (2013). Shifts in methodology and theory in menstrual cycle research on attraction. Sex Roles, 69(9), 525-535. http://dx.doi.org/10.1007/s11199-0130302-3

Havlicek, J., Roberts, S.C. \& Flegr, J. (2005). Women's preference for dominant male odour: effects of menstrual cycle and relationship status. Biology Letters, 1(3), 256-259. http://dx. doi.org/10.1098/rsbl.2005.0332

INJUV. (2017). VIII Encuesta Nacional de Juventud 2015 [VIII Youth National Survey 2015]. Santiago. Chile. Recuperado el 18 de julio de 2017 de http:/www.injuv.gob.cl/portal/wp-con tent/ file s_mf/septima encuestanacional juven tud2.pdf

Instituto Nacional de Estadística e Informática. (2015). Encuesta demográfica y de salud familiar, 2014 [Demographic and family health survey, 2014]. Recuperado el 18 de julio de 2017 de https://www.inei.gob.pe/media/Menu Recursivo/publicaciones_digitales/Est/Lib1211 /pdf/Libro.pdf 
Instituto Nacional de Estadística. (2015). Mujeres en Chile y mercado de trabajo. Participación laboral femenina y brechas salariales [Women in Chile and work market. Female labor participation and income gap]. Recuperado el 18 de julio de 2017 de http://www.ine.cl/canales/ch ile_stadistico/estadisticas_sociales_cultural es/genero/pdf/participacion_laboral_fe

Lehrer, E.L., Lehrer, V.L. \& Krauss, R.C. (2009). Religion and intimate partner violence in Chile: Macro- and micro-level influences. Social Science Research, 38(3), 635-643. http://dx.doi.org/ 0.1016/j.ssresearch.2009.03.001

Little, A.C., Jones, B.C. \& Burriss, R.P. (2007). Preferences for masculinity in male bodies change across the menstrual cycle. Hormones and Behavior, 51(5), 633-639. http://dx.doi.org/ 10.1016/j.yhbeh.2007.03.006

Little, A.C., Jones, B.C. \& DeBruine, L.M. (2008). Preferences for variation in masculinity in real male faces change across the menstrual cycle: Women prefer more masculine faces when they are more fertile. Personality and Individual Differences, 45(6), 478-482. http://dx.doi.org/10. 1016/j.paid.2008.05.024

Little, A.C., Jones, B.C., Penton-Voak, I.S., Burt, D.M. \& Perrett, D.I. (2002). Partnership status and the temporal context of relationships influence human female preferences for sexual dimorphism in male face shape. Proceedings of the Royal Society B: Biological Sciences, 269 (1496), 1095-1100. http://dx.doi.org/10.1098/ rspb.2002.1984

Macintyre, A.K., Montero Vega, A.R. \& Sagbakken, M. (2015). ¿Sexualidad? Un millón de cosas vienen a la mente: Reflexiones sobre género y sexualidad de adolescentes chilenos [Sexuality? A million things come to mind: Reflections on gender and sexuality by Chilean adolescents]. Reproductive Health Matters, 23(46), 85-95. http://dx.doi.org/10. 1016/j.rhm.20 15.11.003.
Miller, G., Tybur, J.M. \& Jordan, B.D. (2007). Ovulatory cycle effects on tip earnings by lap dancers: Economic evidence for human estrus? Evolution and Human Behavior, 28(6), 375381. http://dx.doi.org/10.1016/j.evolhumbehav. 2007.06.002

MINEDUC. (2008). Indicadores de la educación en Chile 2007-2008 [Indicators of the education in Chile 2007-2008]. Recuperado el 18 de julio de 2017 de http://www.scielo. cl/pdf/rmc/ v144n2/art06.pdf

Morán Faúndes, J.M. (2013a). Feminismo, Iglesia Católica y derechos sexuales y reproductivos en el Chile post-dictatorial [Feminism, Catholic Church and sexual and reproductive rights in post-dictatorial Chile]. Estudos Feministas, 21 (2), 485-508.

Morán Faúndes, J.M. (2013b). La anticoncepción de emergencia en Chile: Estructuración de su demanda en función de variables socioeconómicas [Emergency contraception in Chile: Structuring of its demand based on socioeconomic variables]. Revista Brasileira de Estudos de População, 30(1), 125-144.

Muñoz-Reyes, J.A., Iglesias-Julios, M., MartínElola, C., Losada-Pérez, M., Monedero, I., Pita, M. \& Turiégano, E. (2014). Changes in preference for male faces during the menstrual cycle in a Spanish population. Anales de Psicologia / Annals of Psychology, 30(2), 667-675. http://dx. doi.org/10.6018/analesps.30.2.145221

Oinonen, K.A. \& Mazmanian, D. (2002). To what extent do oral contraceptives influence mood and affect? Journal of Affective Disorders, 70 (3), 229-240. http://dx.doi.org/10.1016/S01650327(01)00356-1

Penton-Voak, I.S. \& Perrett, D.I. (2000). Female preference for male faces changes cyclically: Further evidence. Evolution and Human Behavior, 21(1), 39-48. http://dx.doi.org/10.1016/ S1090-5138(99)00033-1 
Penton-Voak, I.S., Perrett, D., Castles, D.L., Kobayashi, T., Burt, D.M., Murray, L.K. \& Minamisawa, R. (1999). Menstrual cycle alters face preference. Nature, 399(6738), 741-742.

Pipitone, R.N. \& Gallup Jr, G.G. (2008). Women's voice attractiveness varies across the menstrual cycle. Evolution and Human Behavior, 29(4), 268-274. http://dx.doi.org/10.1016/j.evolhum be hav.2008.02.001

Roberts, S.C., Havlicek, J., Flegr, J., Hruskova, M., Little, A.C., Jones, B.C. et al. (2004). Female facial attractiveness increases during the fertile phase of the menstrual cycle. Biology Letters, 271(Suppl 5), 270-272. http://dx.doi.org/10.10 98/rsbl.2004.0174

Roberts, S.C., Klapilová, K., Little, A.C., Burriss, R.P., Jones, B.C., DeBruine, L.M. et al. (2012). Relationship satisfaction and outcome in women who meet their partner while using oral contraception. Proceedings of the Royal Society B: Biological Sciences, 279(1732), 1430-1436. http://dx.doi.org/10.1098/rspb.2011.1647

Roberts, S.C., Little, A.C., Burriss, R.P., Cobey, K. D., Klapilová, K., Havlíček, J. et al. (2014). Partner choice, relationship satisfaction, and oral contraception: The congruency hypothesis. Psychological Science, 25(7), 1497-1503. http: //dx.doi.org/10.1177/0956797614532295

Rojas-Barahona, C.A., Zegers, B. \& Förster, C.E. (2009). La Escala de Autoestima de Rosenberg: Validación para Chile en una muestra de jóvenes adultos, adultos y adultos mayores [Rosenberg self-esteem scale: Validation in a representative sample of Chilean adults]. Revista Médica de Chile, 137(6), 791-800. http://dx. doi.org/10.4067/S0034-98872009000600009

Rosenberg, M. (1973). La autoimagen del adolescente y la sociedad [Adolescent's self-image and society]. Buenos Aires: Paidós.

Rosenberg, M.J. \& Waugh, M.S. (1998). Oral contraceptive discontinuation: A prospective evaluation of frequency and reasons. American
Journal of Obstetrics and Gynecology, 179(3), 577-582. http://dx.doi.org/10.1016/S0002-93 78(98)70047-X

Rosenberg, M.J., Waugh, M.S. \& Meehan, T.E. (1995). Use and misuse of oral contraceptives: Risk indicators for poor pill taking and discontinuation. Contraception, 51(5), 283-288. http://dx.doi.org/10.1016/0010-7824(95)0007 4-K

Russell, V.M., McNulty, J.K., Baker, L.R. \& Meltzer, A.L. (2014). The association between discontinuing hormonal contraceptives and wives' marital satisfaction depends on husbands' facial attractiveness. Proceedings of the National Academy of Sciences, 111(48), 17081-17086. http://dx.doi.org/10.1073/pnas.1414784111

Sanders, S.A., Graham, C.A., Bass, J.L. \& Bancroft, J. (2001). A prospective study of the effects of oral contraceptives on sexuality and well-being and their relationship to discontinuation. Contraception, 64(1), 51-58. http://dx. doi.org/10.1016/S0010-7824(01)00218-9

Schiappacasse, V., Bascuñan, T., Frez, K. \& Cortés, I. (2014). Píldora anticonceptiva de emergencia: características de la demanda en una organización no gubernamental en Chile [Emergency contraceptive pill: demand characteristics in a non-governmental organization in Chile. Revista Chilena de Obstetricia y Ginecología, 79(5), 378-383. http://dx.doi.org/10.4067/S0717-7526 2014000500004

Schuster, I., Krahé, B., Ilabaca Baeza, P. \& MuñozReyes, J.A. (2016). Sexual aggression victimization and perpetration among male and female college students in Chile. Frontiers in Psychology, 7, 1354. http://dx.doi.org/10.3389/fp syg.2016.01354

Serrano, I. (2009). VI Encuesta Daphne-Bayer Schering Pharma [VI Daphne-Bayer Schering Pharma survey]. Recuperado el 18 de julio de 2017 de http://www.sidastudi.org/ es/registro/d 25f08042de87f95012dea8f6a4a0025. 
Silva, J. \& Barrientos, J. (2008). Guiones sexuales de la seducción, el erotismo y los encuentros sexuales en el norte de Chile [Sex scripts of seduction, eroticism and sexual encounters in northern Chile]. Estudos Feministas, 16(2), 539-556.

Singh, D. \& Bronstad, P.M. (2001). Female body odour is a potential cue to ovulation. Proceedings of the Royal Society of London. Series B: Biological Sciences, 268(1469), 797-801. http: //dx.doi.org/10.1098/rspb.2001.1589

Spencer, A.L., Bonnema, R. \& McNamara, M.C. (2009). Helping women choose appropriate hormonal contraception: Update on risks, benefits, and indications. American Journal of Medicine, 122(6), 497-506. http://dx.doi.org/ 10.1016/j.amjmed.2009.01.016

van Heusden, A.M. \& Fauser, B.C.J.M. (2002). Residual ovarian activity during oral steroid contraception. Human Reproduction Update, 8 (4), 345-358. http://dx.doi.org/10.1093/humup $\mathrm{d} / 8.4 .345$

Walker, A. \& Bancroft, J. (1990). Relationship between premenstrual symptoms and oral contraceptive use: A controlled study. Psychosomatic Medicine, 52(1), 86-96.

Wallwiener, M., Wallwiener, L.M., Seeger, H., Mueck, A.O., Zipfel, S., Bitzer, J. \& Wallwiener, C.W. (2010). Effects of sex hormones in oral contraceptives on the female sexual function score: a study in German female medical students. Contraception, 82(2), 155-159. http:// dx.doi.org/10.1016/j.contraception.2009.12.022

Welling, L.L. M. (2013). Psychobehavioral effects of hormonal contraceptive use. Evolutionary Psychology, 11(3), 718-742. http://dx.doi.org/ 10.1177/147470491301100315

Universidad de Playa Ancha Valparaíso Chile

Fecha de recepción: 24 de mayo de 2016 Fecha de aceptación: 24 de julio de 2017 